\title{
A Study of Possible Solar Sail Applications for Mars Missions
}

\author{
Thomas K. Percy" \\ Science Applications International Corporation, Huntsville, AL, 35806 \\ and \\ Travis Taylor ${ }^{\dagger}$ and T. Conley Powell ${ }^{\ddagger}$ \\ BAE Systems, Huntsville, AL, 35806
}

\begin{abstract}
A study was performed in conjunction with the In Space Technology Investment Area of NASA's Marshall Space Flight Center to investigate potential applications of solar sails to Mars missions. While solar sails have been proposed as possible candidates for several missions, including Geostorm and a Polar Observer mission, Mars has often been overlooked as a potential destination. It was found that solar sails may have potential in Mars observation missions with smaller payloads. Sail aerocapture maneuvers also show an improvement in payload delivery capability. This study has shown that solar sail spacecraft rival chemical interplanetary probes to Mars and may have applications in future Mars exploration.
\end{abstract}

\section{Nomenclature}

$\begin{array}{ll}\beta & =\text { solar sail lightness parameter } \\ \mu & =\text { gravitational parameter } \\ \sigma & =\text { sail film areal density }\end{array}$

\section{Introduction}

A study was performed in conjunction with the In Space Technology Investment Area of NASA's Marshall Space Flight Center to investigate potential applications of solar sails to Mars missions. While solar sails have been proposed as possible candidates for several missions, including Geostorm and a Polar Observer mission, Mars has often been overlooked as a potential destination. Solar sailing has not been extensively considered for Mars in large part because industry is proficient at completing these missions with current chemical propulsion technologies. However, in an effort to investigate new and potentially superior propulsion options for Mars missions, solar sails were studied. Some of the preliminary results will be reviewed here.

Solar sailing presents many possible benefits in the arena of interplanetary exploration. By eliminating virtually all propellant requirements, solar sails present a significant weight reduction over traditional chemical systems. For inner planet missions, where there exists a fairly high level of solar photonic pressure, solar sails can provide maneuvering capabilities once on station around a planet. This orbital flexibility may offer the opportunity to investigate several different destinations at a planet. For example, by carrying a modest instrument suite, a solar sail spacecraft could potentially visit several moons of a single planet with no additional propellant requirement. All that is necessary to complete the maneuver is time.

Mission and systems analysis techniques and assumptions will be outlined in the following pages. Analysis of different trajectories for solar sail travel to Mars will be discussed. The subsystems involved in a solar sail spacecraft will also be outlined and assumptions about functionality and mass will be laid out. Finally a case study involving a small scientific mission to Mars' moon Phobos will be discussed to display one possible application of solar sailing to Mars exploration missions.

\footnotetext{
- Systems Engineer, System Technologies, SAIC, 675 Discovery Dr. Suite 300, Huntsville, AL 35806, Member.

+ Senior Scientist, Advanced Projects, BAE Systems, 310 Voyager Way, Huntsville, Alabama 35806

${ }^{\ddagger}$ Senior Scientist, Advanced Projects, BAE Systems, 310 Voyager Way, Huntsville, Alabama 35806.
} 


\section{Analysis Overview}

The analyses that comprised this study were broken down into two separate but related areas. The first involved mission analyses performed in the areas of trajectory and general mission architecture. A conceptual systems integration model facilitated the systems interaction analyses performed in the second area of the study. This model allowed analyses on subsystem interactions with emphasis on subsystem mass, a critical characteristic in solar sail design. By coupling these two analysis regimes, a fairly complete initial analysis was performed which allowed the tracking of mission architecture affects on system and subsystem performance.

Several assumptions were first laid out to simplify the analysis. Specific assumptions will be discussed in detail in the systems analysis segment of this report. One important universal assumption involves mass margins for the analysis. A margin of $30 \%$ was added to all subsystem component masses. This conservative estimate allows for growth from the current best estimates of subsystem masses that are available from industry. While a complete solar sail spacecraft design is highly dependent on the mass of the system, and thus may be penalized by overly conservative estimations, it was the consensus of the parties involved that current mass estimates were far too speculative to allow for smaller margins.

Figures of merit for the analysis included transfer time and launch vehicle requirements. Typical solar sail designs offer a reduction in launch mass that may lead to the use of smaller and less costly launch vehicles. While cost is not specifically addressed, launch vehicle selection was considered for this study to reflect this potential benefit. The main driver of the study was the investigation of overall payload delivery capability of the solar sail system, both in general and as specifically applied to the Phobos DRM.

\section{Mission Analysis}

\section{A. Earth-Mars Trajectory Analysis}

The mission analyses performed for this study involved trajectory analysis ${ }^{1,4,8}$. Various mission designs for sending solar sail spacecraft to a closed Mars orbit from Earth orbit were presented. The mission trajectory types considered were simple ballistic trajectories with radial and asymptotic thrust vectors, Hohmann-A transfers, and the Sundiver transfer ${ }^{8}$. The results obtained suggest that the ballistic trajectory makes the most sense for an Earth-toMars solar sail mission. The Sundiver mission approach offers no advantage.

It was necessary to make a few approximations and assumptions for the trajectory analysis. The patched-conic approximation was used and it was assumed that the orbits of Earth and Mars are circular and coplanar. Also, other body influence such as the outer or inner planets were not modeled, as their influence should be minimal for this type of mission.

The approach taken in this analysis utilized modified orbital equations based on the effective gravitational constant, $(1-\beta) \mu$, where $\beta$ is the lightness parameter of the solar sail spacecraft and $\mu$ is the gravitational constant of the $\operatorname{sun}^{8}$. The angle $\alpha$ was defined as the angle between the asymptotic velocity vector and Earth's radius vector and allowed for the tracking of the solar sail's attitude with respect to the solar pressure. This angle was held constant for simplicity of analysis.

As seen in Figures 1 and 2, the trip time is significantly reduced by opting for a direct transfer as opposed to the Sundiver maneuver which is popular with the solar sailing community for outer planet missions. These charts assume an $\alpha$ of $90^{\circ}$ and an Earth escape velocity of $3000 \mathrm{~m} / \mathrm{s}$.

From the derived trajectory data, Mars arrival conditions were determined and capture methods were investigated. The hyperbolic approach velocity at Mars was found to be on the order of 5 to $12 \mathrm{~km} / \mathrm{s}$. A maneuver which will be referred to as sail aerocapture was considered as the method of braking at Mars ${ }^{12}$. Essentiaily, by passing through the outer levels of the appreciable Martian atmosphere, the large sail area would provide enough drag to reduce the velocity and capture into a highly elliptical orbit. Once this capture maneuver is completed, a small thruster would raise the periapsis of the orbit out of the atmosphere and

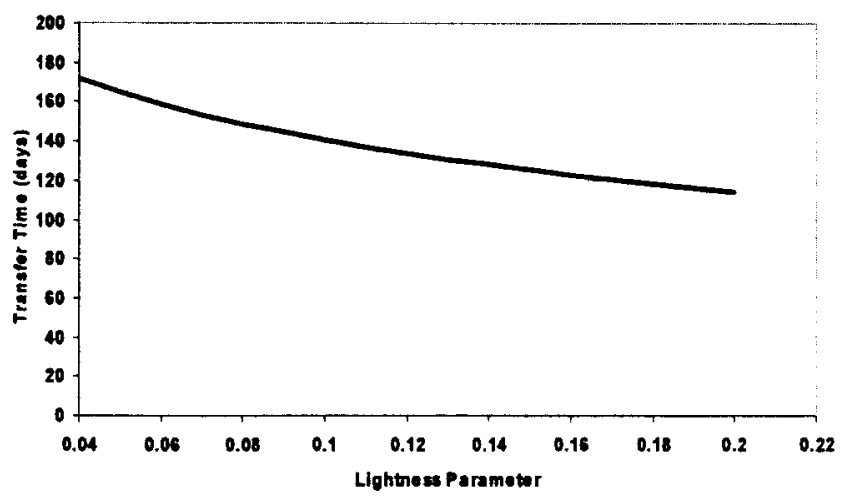

Figure 1: Trip Time versus Lightness Parameter; Earth Escape velocity equals $3000 \mathrm{~m} / \mathrm{s}$ 
solar pressure would be used by the sail craft to maneuver into the final orbit around Mars. This maneuver is currently under investigation, though initial indications are that it will be possible. More will be shown about the different capture techniques investigated in the systems analysis section.

\section{B. Orbit Raising at Mars}

Maneuvering in the Martian system to raise the orbit of the solar sail will take anywhere from 30 to 70 days based on preliminary models developed for this study. The maximum light-pressure acceleration obtainable from the solar sail is $\beta \mu_{\mathrm{S}} / r_{\mathrm{M}}{ }^{2}$, where

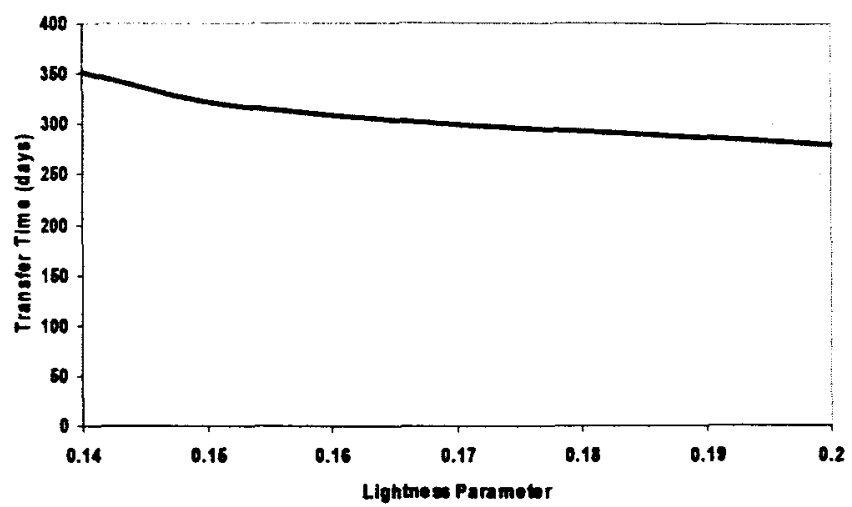

Figure 2: Trip Time versus Lightness Parameter; Earth Escape velocity equals $3000 \mathrm{~m} / \mathrm{s}$; Sundiver Maneuver $\mu_{\mathrm{S}}$ is the gravitational constant for the Sun and $r_{\mathrm{M}}$ is the radius of Mars' orbit. $\mu_{\mathrm{S}} / r_{\mathrm{M}}{ }^{2}$ is $2.5546 \times 10^{-3} \mathrm{~m} / \mathrm{s}^{2}$. Thus the maximum light-pressure acceleration for a vehicle with a lightness parameter of 0.2 is $5.1092 \times 10^{-4} \mathrm{~m} / \mathrm{s}^{2}$. The maximum change in orbital radius per revolution is given approximately by

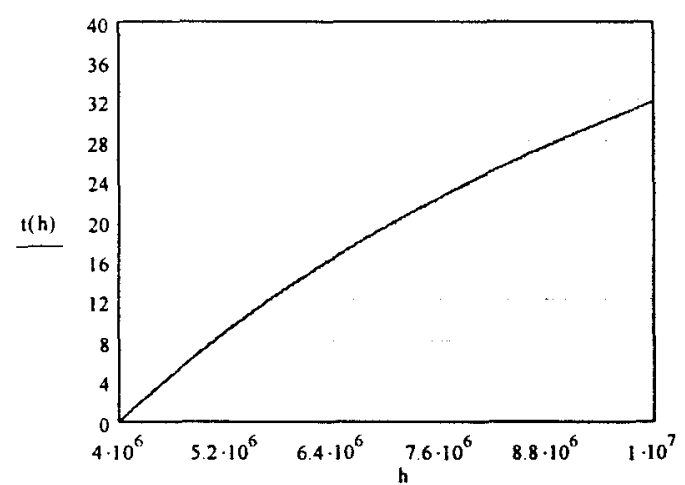

Figure 3: Time for Orbit Raising

$$
\Delta r=5.52 \beta \mu_{S} r^{3} / \mu_{M} r_{M}^{2}
$$

from which the equation

$$
\left(\frac{\mu_{M}}{r}\right)-\left(\frac{\mu_{M}}{r_{0}}\right)=\frac{1.38 \beta \mu_{S} t}{p r_{M}^{2}}
$$

is obtained in which $r_{0}$ is the initial radius at time $t=0$.

Figure 3 shows the results of orbit raising from a circular orbit at a height of $4,000 \mathrm{~km}$ to a final height of $10,000 \mathrm{~km}$ using a solar pressure propelled, spiral trajectory for orbit raising. The orbit height is given as a function of time. The reduced solar pressure is still sufficient for orbital flexibility with a solar sail spacecraft.

This orbital flexibility allows for investigation of Martian satellites and the ability to modify Mars orbits for coverage of a wide range of Martian latitudes and longitudes.

\section{System Analysis}

The system analysis approach taken in this study facilitated the assessment of subsystem masses and the effect of subsystem interactions on that mass. The systems unique to solar sail spacecraft were assessed based on industry estimations and projections of fabrication capabilities. Subsystems which are similar to those used in current interplanetary probes are scaled from existing systems. In many cases these subsystems were scaled from the Mars Odyssey spacecraft launched in 2001. A schematic of the solar sail system can be seen in figure 4 .

\section{A. Solar Sail Systems}

Systems unique to solar sail spacecraft are focused mainly in propulsion, specifically the sail and sail structure and the attitude control system. Sail shape was restricted to a square configuration to follow with the current solar sail investigations taking place in the field. In addition, CPI was selected as the baseline sail material. This material is currently under examination in several solar sail test cases ${ }^{7,9}$. In addition to its use in solar sail scale modeling, the fabrication of large sheets of CP1 is also being investigated. This leads to the conclusion that a square sail comprised of sheets of CP1 is the most likely candidate for first generation solar sails. For this study, the system was assessed parametrically over a range of areal densities from 2 to $10 \mathrm{~g} / \mathrm{m}^{2}$ and a reflectivity of $85 \%$ was assumed to account for any degradation over the life of the system. 
The sail structure was assumed to be a deployable boom system. There are two major designs for deployable boom structures currently being considered for solar sails ${ }^{7,10}$. Both designs require little or no deployment mechanisms. The linear density of the boom structure for this study was assumed to be $50 \mathrm{~g} / \mathrm{m}$ based on industry projections.

Attitude control of solar sail spacecraft can be achieved with several different types of systems ${ }^{8}$. For this model, a payload boom with a gimbal system was assumed for shifting the center of gravity of the system with respect to the center of pressure. This allows for directional control during the

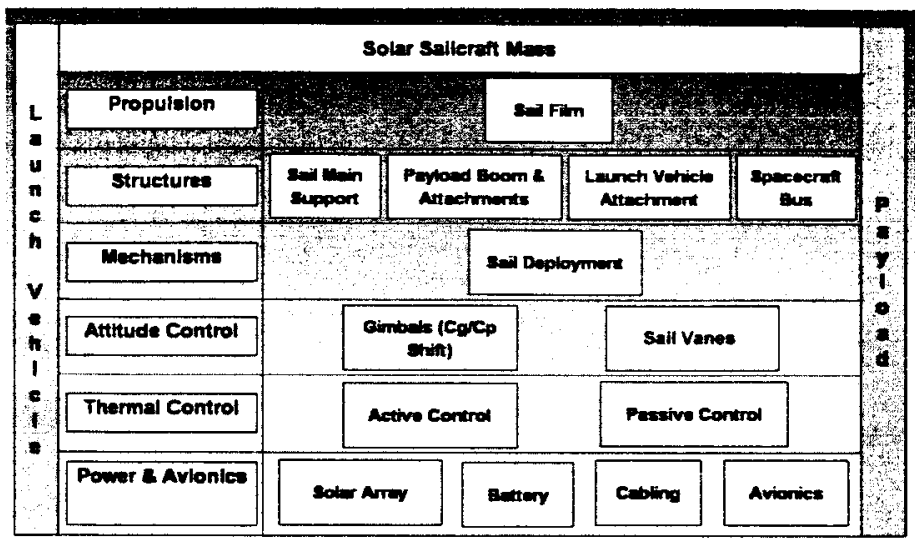

Figure 4: Solar sail spacecraft subsystem breakdown Earth-Mars portion of the solar sail trajectory and may also prove useful during the sail aerocapture maneuver at Mars. Small reflective structures known as sail vanes were also included at the tips of the sail booms for quick response attitude control during the orbit raising portion of the solar sail function. For mass estimation purposes the sail vanes are assumed to be $0.5 \%$ of the total sail area. Small reaction wheels were also included for spacecraft stabilization during the sail deployment operations.

\section{B. Spacecraft Bus and Other Subsystems}

The spacecraft bus was assumed to be a highly integrated system at the end of the payload boom, thus facilitating a traditional analysis approach to subsystems such as power, data handling, navigational instrumentation, thermal control and structural mass. The structure of the bus is based on historical data from other satellite programs and interplanetary probes. Given that the bus structure for the solar sail spacecraft is slightly smaller than typical interplanetary probes, the model was verified by cross referencing with micro-satellite structure masses. The launch vehicle adapter and attachments were assumed to be $55 \mathrm{~kg}$ and these structures were assumed to be jettisoned before transit and thus not included in the spacecraft mass for trajectory assessment ${ }^{31}$.

The power supply for the spacecraft was sized based on $115 \mathrm{~W} / \mathrm{kg}$ solar arrays. The power requirement for the spacecraft subsystems was assumed to be $1.5 \mathrm{~kW}$ EOL at $1 \mathrm{AU}$. Batteries with a mass of $10 \mathrm{~kg}$ were also included for power storage. The mass of the navigation and avionics systems was taken directly from the systems on Mars Odyssey.

\section{Capture Method Analysis}

A simple trade of capture technique was performed taking two procedures into consideration. The first was the previously mentioned sail aerocapture, which requires a very small chemical system for raising the periapsis of the

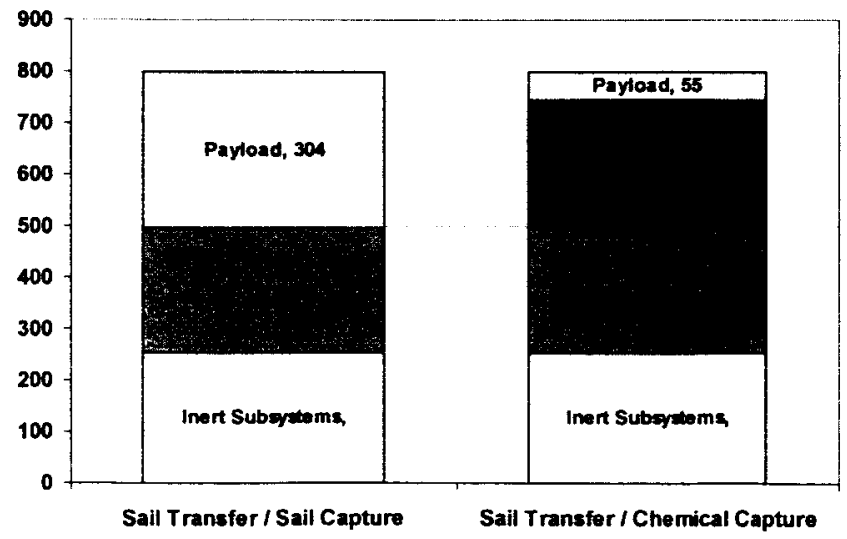

Figure 5: Stack Masses for Capture Mode Comparison initial orbit of the solar sail. This system was included at a mass of approximately $10 \mathrm{~kg}$. The second option was the traditional chemical burn for orbital insertion. The results show that carrying the required chemical capture system limits the payload capability. This technique also increases the overall sail size necessary to complete the mission due to the increase in overall spacecraft mass. In some cases the mass was increased by as much as $225 \%$ over the sail aerocapture system. For example, results show that the sail size would be $20,705 \mathrm{~m}^{2}$ with a chemical capture system compared to 10,870 $\mathrm{m}^{2}$ in the aerocapture mode. Also, chemical capture may require the jettisoning of the sail system before the capture burn in order to reduce propellant requirements and allow the 
spacecraft to be launched on a Delta II as opposed to a Delta IV launch vehicle 5 . The analysis results indicate that choosing a chemical capture system puts a limit on potential scientific payload that is only slightly higher than the DRM payload where the selection of aerocapture allows significantly higher payload delivery (see Figure 5). Thus it can be seen that sail aerocapture has significant potential for increasing mission performance.

In a comparison of scientific payload delivery versus transfer time, it is shown that increasing the lightness number will reduce the trip time however it also reduces the mass delivery potential if the total spacecraft mass is held constant. Given a

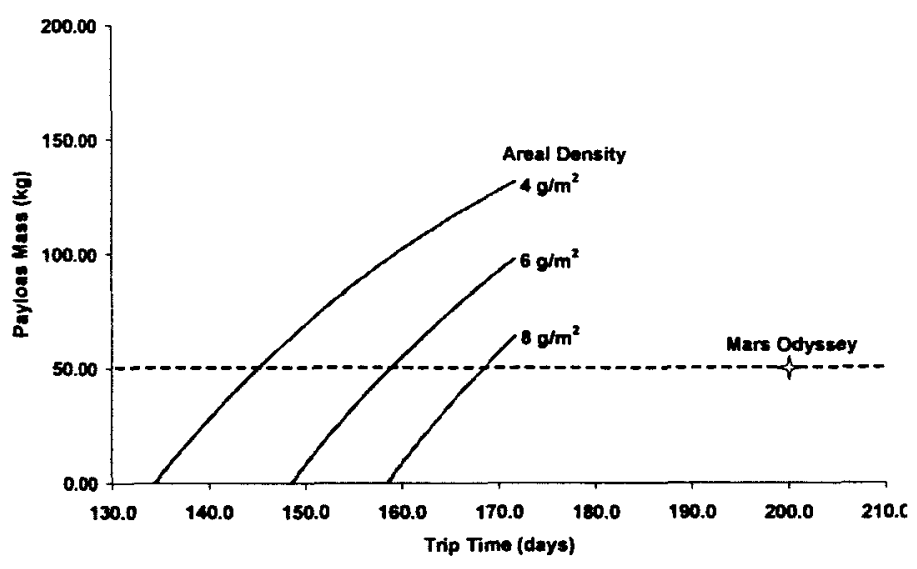

Figure 6: Sail payload delivery potential versus trip time spacecraft mass, a trade must be made between sail mass (increased sail size reduces transfer time) and payload delivery mass capability. Figure 6 presents this finding as well as a comparison to the Mars Odyssey mission. Both the solar sail spacecraft and the Mars Odyssey have the same dry spacecraft mass if their respective propulsion system masses are ignored. Not only can the solar sail deliver more scientific payload mass than was delivered by Mars Odyssey, but it is also capable of doing so in a shorter amount of time.

\section{Case Study: Phobos Rendezvous}

For this study, a specific reference mission was also investigated. The mission entailed delivering a $50 \mathrm{~kg}$ instrument suite to an orbit that matches the Martian moon Phobos for the purpose of scientific observation. The Mars Odyssey spacecraft's scientific instrument suite, weighing approximately $50 \mathrm{~kg}$, was chosen as a model for this mission.

The solar sail system was modeled parametrically over a range of sail film areal densities to investigate the effect of further development of sail materials. Taking into account the system analysis assumptions mentioned above, the analysis was run with the goal of delivering a scientific instrument suite of $50 \mathrm{~kg}$. Figure 7 displays the payload delivery capability of a $500 \mathrm{~kg}$ solar sail spacecraft over a range of trip times. The figure shows that as trip time increases so too does the payload capability. This is due to the fact that increased trip time equates to a reduced sail area for a standard spacecraft mass. This reduction in sail area reduces the mass of the sail dependent systems (solar sail film, sail structure etc.) and provides more margin for payload mass. Figure 7 also displays the benefit of lower film areal densities.

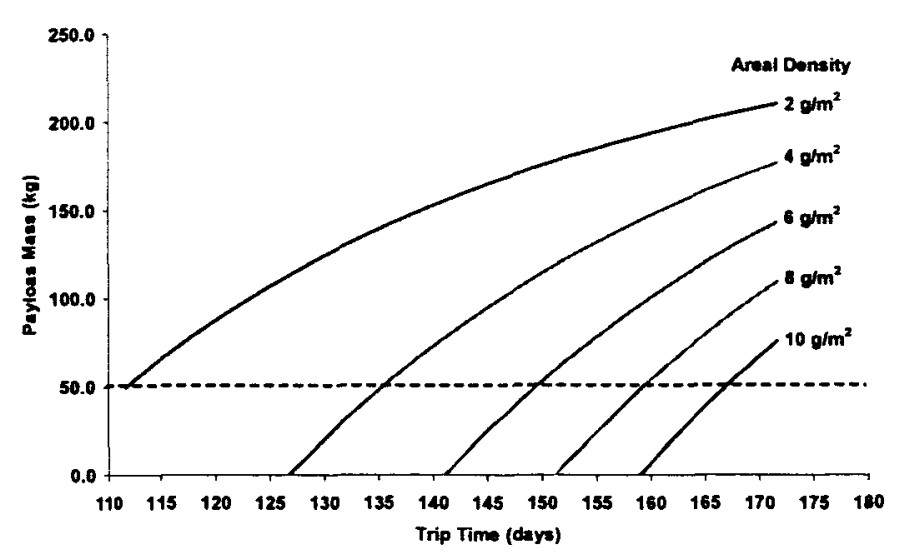

Figure 7: Sail payload delivery potential versus trip time over a range of film areal densities 
Table 1: Subsystem Masses

\begin{tabular}{|lr|}
\hline Power System & $62.2 \mathrm{~kg}$ \\
Sail Film & $189.3 \mathrm{~kg}$ \\
Sail Structure & $22.2 \mathrm{~kg}$ \\
S/C Bus & $31.6 \mathrm{~kg}$ \\
Hardware \& Attac & $24.6 \mathrm{~kg}$ \\
TPS & $28.6 \mathrm{~kg}$ \\
ACS & $20.4 \mathrm{~kg}$ \\
Avionics & $53.3 \mathrm{~kg}$ \\
Scientific Payload & $50.2 \mathrm{~kg}$ \\
PR Propulsion & $9.7 \mathrm{~kg}$ \\
Other & $7.8 \mathrm{~kg}$ \\
& 500.0 \\
\hline
\end{tabular}

In one specific case, a $500 \mathrm{~kg}$ spacecraft system can deliver a $50 \mathrm{~kg}$ payload in 168 days with a sail area of $14,558 \mathrm{~m}^{2}$, given a sail lightness parameter of 0.045 and a sail areal density of 10 $\mathrm{g} / \mathrm{m}^{2}$. The characteristic acceleration of this system is 0.226 $\mathrm{mm} / \mathrm{s}^{2}$. The mass of the subsystems of this solar sail spacecraft can be seen in Table 1. This system can be launched in a Delta II launch vehicle ${ }^{5}$.

\section{Conclusion}

It can be seen from this study that solar sails have the potential for missions to Mars if the technology develops as projected. Their mass delivery potential rivals that of previous scientific observation missions. Transfer times calculated with systems employing solar photonic pressure are shorter than most Hohmann Type I trajectories currently used by conventional chemical systems. The trajectory models also indicated that a departure from conventional solar sail Sundiver maneuver is necessary to bring transfer times to competitive levels in Earth-to-Mars situations. There is a potential for orbital flexibility on station that could allow more than one mission to be performed by one orbiting platform. Also, the projected reductions in injected mass could reduce overall mission cost by allowing for the use of smaller launch vehicles.

Finally, the issue of capture technique at Mars was addressed. Study results indicate that sail aerocapture is an effective technology that, could impact a range of solar sail missions. Using a solar sail transfer vehicle and capturing with conventional chemical propulsion systems vastly reduces the effectiveness of the solar sail technology. However, by integrating aerocapture technology, the full potential of the solar sail spacecraft can be realized. While solar sails are effective for missions like Geostorm and Polar Observer, this study shows the potential for solar sail application to a much broader portfolio of missions.

\section{Acknowledgments}

The work described in this paper was performed by Science Application International Corporation (SAIC) and BAE Systems under contract with the NASA Marshall Space Flight Center (MSFC) in Huntsville, Alabama. Special thanks go to Les Johnson, manager of NASA MSFC In-Space Propulsion, and Sandy Montgomery, Solar Sail technology area manager, for providing encouragement and direction for this work. T.K. Percy would like to thank his wife, Erin, for her patience and support. T.K. Percy would like to give special recognition to SAIC management, especially Frank Curran, program manager of the In-Space Technology Assessment program, for support and encouragement of this work and to Mike Cupples for his guidance. T. C Powell would like to acknowledge the patience and support of his wife, Judy. 


\section{References}

'Bate, R., Mueller, B., White, J. Fundamentals of Astrodynamics. Dover Publications, Inc. New York, 1971.

${ }^{2}$ Frisbee, R. , Brophy, J. "Inflatable Solar Sails for Low-Cost Robotic Mars Missions". AIAA 97-2762.

${ }^{3}$ Garner C. et al. "Developments and Activities in Solar Sail Propulsion". AIAA 2001-3234.

${ }^{4}$ George, L.E. , Kos, L.D. "Interplanetary Mission Design Handbook: Earth to Mars Opportunities and Mars to Earth Opportunities, 2009-2024". NASA/TM 1998-208533.

${ }^{5}$ Isakowitz, S.J. et al. International Reference Guide to Space Launch Systems. $3^{\text {rd }}$ ed., AIAA, Reston, 1996.

${ }^{6}$ Leipold, M. et al. "Solar Sail Technology Development and Demonstration". 2000.

${ }^{7}$ Lichodziejewski, D. et al. "Bringing an Effective Solar Sail Design Toward TRL 6". AlAA 2003-4659.

${ }^{8}$ McInnes, C. Solar Sailing: Technology, Dynamics and Mission Applications. Springer Verlag 1998.

${ }^{9}$ Murphy, D. et al. "Progress And Plans For System Demonstration of a Scalable Square Sail System". AIAA/ASME/SAE/ASEE Joint Propulsion Conference, 2003.

${ }^{10}$ Murphy, D. et al. "Scalable Solar Sail Subsystem Design Considerations". AIAA 2002-1703.

"Noca, M. et al. "Evaluating Advanced Propulsion Systems for the Titan Explorer Mission". IEPC-01-175.

${ }^{12}$ Taylor, T. et al. "Solar Sail Application to Comet Nucleus Sample Return”. AIAA 2003-5275. 